\title{
Estimated Dietary Polyphenol Intake and Major Food and Beverage Sources among Elderly Japanese
}

\author{
Chie Taguchi $^{1}$, Yoichi Fukushima ${ }^{2}$, Yoshimi Kishimoto ${ }^{1, *}$, Norie Suzuki-Sugihara ${ }^{3}$, Emi Saita ${ }^{1}$,
} Yoshinari Takahashi ${ }^{4}$ and Kazuo Kondo ${ }^{1,5}$

Received: 9 November 2015; Accepted: 2 December 2015; Published: 9 December 2015

1 Endowed Research Department "Food for Health", Ochanomizu University, 2-1-1 Otsuka, Bunkyo-ku, Tokyo 112-8610, Japan; taguchi.chie@ocha.ac.jp (C.T.); saita.emi@ocha.ac.jp (E.S.);

kondo.kazuo@ocha.ac.jp (K.K.)

2 Nestlé Japan Ltd., NYK Tennoz Blidg., 2-2-20 Higashi-Shinagawa, Shinagawa-ku, Tokyo 140-0002, Japan; yoichi.fukushima@jp.nestle.com

3 Department of Nutrition and Food Science, Graduate School of Humanities and Sciences, Ochanomizu University, 2-1-1 Otsuka, Bunkyo-ku, Tokyo 112-8610, Japan; g1370512@edu.cc.ocha.ac.jp

4 TES Holdings Co., Ltd., 6F Tokyo University Entrepreneurs Plaza, 7-3-1 Hongo, Bunkyo-ku, Tokyo 113-0033, Japan; y.takahashi@tes-h.co.jp

5 Institute of Life Innovation Studies, Toyo University, 1-1-1 Izumino, Itakura-machi, Ora-gun, Gunma 374-0193, Japan

* Correspondence: kishimoto.yoshimi@ocha.ac.jp; Tel.: +81-359-785-810; Fax: +81-359-782-694

\begin{abstract}
Estimating polyphenol intake contributes to the understanding of polyphenols' health benefits. However, information about human polyphenol intake is scarce, especially in the elderly. This study aimed to estimate the dietary intake and major sources of polyphenols and to determine whether there is any relationship between polyphenol intake and micronutrient intake in healthy elderly Japanese. First, 610 subjects (569 men, 41 women; aged $67.3 \pm 6.1$ years) completed food frequency questionnaires. We then calculated their total polyphenol intake using our polyphenol content database. Their average total polyphenol intake was $1492 \pm 665 \mathrm{mg} /$ day, the greatest part of which was provided by beverages (79.1\%). The daily polyphenol intake differed largely among individuals (183-4854 mg/day), also attributable mostly to beverage consumption. Coffee $(43.2 \%)$ and green tea $(26.6 \%)$ were the major sources of total polyphenol; the top 20 food items accounted for $>90 \%$. The polyphenol intake did not strongly correlate with the intake of any micronutrient, suggesting that polyphenols may exert health benefits independently of nutritional intake. The polyphenol intake in this elderly population was slightly higher than previous data in Japanese adults, and beverages such as coffee and green tea contributed highly to the intake.
\end{abstract}

Keywords: polyphenol; consumption; beverage; food; elderly; coffee; green tea

\section{Introduction}

Polyphenols are present in high amounts in most plant foods and beverages [1], and cannot be synthesized by humans [2]. In the 1990s, several epidemiological studies demonstrated that dietary polyphenol consumption is associated with a reduced risk of cardiovascular disease [3,4]. As the basic and clinical research progressed, multiple functions of polyphenols contributing to human health were identified $[5,6]$. To elucidate the contribution of polyphenols to human health, it is necessary to estimate individuals' polyphenol intake in their daily diet. Comprehensive databases of the polyphenol content of food such as the United States Department of Agriculture (USDA) Database for the Flavonoid Content of Selected Foods [7] and the Phenol-Explorer databases [8,9] by the French National Institutes for Agricultural Research have been established and used for estimating the individuals' polyphenol intake in European and American countries [10-13]. Through the estimation 
of dietary polyphenols, several cohort studies have shown an inverse association between polyphenol intake and the risk of chronic diseases such as cardiovascular diseases [14-20], cancers [21-25], and all-cause mortality [26]. In a recent publication from the PREDIMED trial, a 37\% reduction of mortality was observed in a comparison of extreme quintiles of total polyphenol intake [27]. However, information about polyphenol intake in Japanese populations is still limited [28].

Japan has a unique food culture, and thus a database on the polyphenol contents of foods consumed in Japan is needed to estimate the daily polyphenol intake in Japanese. We measured total polyphenol contents by conducting a modified Folin-Ciocalteu colorimetric assay for 77 food and beverage items, and established a database for the polyphenol content. Using the database, we have assessed the daily polyphenol intake in the following Japanese populations: (1) randomly selected male and female subjects (10-59 years old, $n=8768)$ who recorded all non-alcoholic beverages consumed in a week [29]; (2) 109 middle-aged Japanese women who recorded all beverages and foods ingested in a week [30]; and (3) randomly selected male and female subjects (1-99 years old, $n>10,000$ every year for 18 years) who recorded all beverages consumed in a week [31]. According to these studies, we documented that coffee and green tea are the major contributors to total polyphenol consumption [29-31]. However, there is very limited information about the dietary polyphenol intake among elderly Japanese.

The elderly population has been growing rapidly both in Japan and worldwide. Polyphenols have been recognized to be beneficial for elderly people, to prevent chronic diseases and maintain health. It was recently reported that dietary polyphenol might be associated with lower risks of substantial cognitive decline [32] and frailty [33] in older adults. A polyphenol-rich diet also has potential for increasing specific nutrients, because plant foods and beverages are also the main sources of vitamins, minerals or fiber in the diet. However, the relationship between dietary polyphenol and nutrients has not been elucidated.

This study was conducted to (1) estimate the polyphenol intake among elderly Japanese; (2) determine the contributions of specific foods to the total polyphenol intake; and (3) clarify the relationships between polyphenol and micronutrient intake.

\section{Experimental Section}

\subsection{Study Population}

We posted two questionnaires by mail to 986 Japanese listed as retired employees of Nestlé Japan Ltd., and from 685 replies we obtained 627 subjects who completed two questionnaires. We excluded 17 subjects whose energy intake was either too low (at less than one-half the energy requirement for the lowest physical activity category) or too high (at 1.5 times more than the energy requirement of the highest physical activity category), referring to the Dietary Reference Intakes for Japanese (2010) [34]. Thus, 610 subjects ( 569 men and 41 women) aged 52-89 (67.3 \pm 6.1 years) were included in this analysis.

\subsection{Estimation of Dietary Polyphenol}

The subjects' dietary habits during the previous month were assessed using two different food frequency questionnaires (FFQs). One was our originally developed FFQ for polyphenol intake from food and beverages that assesses the intake of 22 foods and 23 beverages, which are significant items rich in polyphenol according to our previous studies $[29,30]$. The other FFQ was a brief-type self-administered diet history questionnaire (BDHQ), which assesses the intake frequency of 58 food and beverage items $[35,36]$. Each subject's consumption of each food item was obtained using the two FFQs. The intakes of energy and nutrients were estimated using the intakes of food items described in the BDHQ. The subjects filled out the two FFQs in September or October of 2012.

In this study, we used our original database of the polyphenol content of 67 items: 15 types of beverages and 52 types of foods, mostly described in previous reports [29,30]. The polyphenol contents of chocolate were revised as $390 \mathrm{mg} / 100 \mathrm{~g}$ and $1560 \mathrm{mg} / 100 \mathrm{~g}$, respectively, for milk 
chocolate (5\% cacao mass) and bitter chocolate $(20 \%$ cacao mass) from the previous values ( $935.5 \mathrm{mg} / 100 \mathrm{~g}$ for $12 \%$ cacao mass) [30]. We calculated the polyphenol intake by matching the food consumption data from two FFQs with the polyphenol content in the food and beverages described above.

\subsection{Statistical Analysis}

All data are expressed as mean \pm standard deviation (SD). Statistical analyses were performed with IBM SPSS software, version 20. Spearman's correlation coefficients were calculated to assess the relationships between variables.

\section{Results}

\subsection{Subjects' Characteristics}

The characteristics of the 610 subjects are shown in Table 1. As noted above, the mean age of the 569 men and 41 women was $67.3 \pm 6.1$ years (range $52-89$ years). The average body mass index (BMI) was $23.1 \pm 2.6 \mathrm{~kg} / \mathrm{m}^{2}$ in the men and $22.3 \pm 2.8 \mathrm{~kg} / \mathrm{m}^{2}$ in the women. The estimated daily energy was $2185 \pm 594 \mathrm{kcal} /$ day in the men and $1670 \pm 475 \mathrm{kcal} /$ day in the women. The average dietary energy and nutrient intakes were almost the same as the average Japanese intakes referred to in a recent National Health and Nutrition Survey [37] and were of almost sufficient quantity to meet the Adequate Intake of Dietary Reference Intakes for Japanese (2010) [34].

Table 1. Characteristics of the population (569 men and 41 women) and their nutritional intake.

\begin{tabular}{cccccccc}
\hline & & \multicolumn{2}{c}{ Men and Women $(\boldsymbol{n}=\mathbf{6 1 0})$} & \multicolumn{2}{c}{ Men $(\boldsymbol{n}=\mathbf{5 6 9})$} & \multicolumn{2}{c}{ Women $(\boldsymbol{n}=\mathbf{4 1})$} \\
\cline { 3 - 8 } & & Mean \pm SD & Min-Max & Mean \pm SD & Min-Max & Mean \pm SD & Min-Max \\
\hline Age & years & $67.3 \pm 6.1$ & $(52-89)$ & $67.3 \pm 6.0$ & $(55-89)$ & $66.7 \pm 7.2$ & $(52-81)$ \\
Body weight & $\mathrm{kg}$ & $64.3 \pm 8.8$ & $(35.0-95.0)$ & $65.0 \pm 8.4$ & $(46.0-95.0)$ & $54.0 \pm 8.0$ & $(35.0-69.0)$ \\
BMI & $\mathrm{kg} / \mathrm{m}^{2}$ & $23.1 \pm 2.7$ & $(15.1-34.5)$ & $23.1 \pm 2.6$ & $(16.6-34.5)$ & $22.3 \pm 2.8$ & $(15.1-28.6)$ \\
Energy & $\mathrm{kcal}$ & $2150 \pm 600$ & $(895-4035)$ & $2185 \pm 594$ & $(1031-4035)$ & $1670 \pm 475$ & $(895-2898)$ \\
Protein & \% energy & $15.5 \pm 3.1$ & $(8.9-28.1)$ & $15.4 \pm 3.1$ & $(8.9-28.1)$ & $16.9 \pm 3.1$ & $(10.7-24.3)$ \\
Fat & \% energy & $26.4 \pm 5.1$ & $(14.5-43.5)$ & $26.2 \pm 5.1$ & $(14.5-43.5)$ & $29.4 \pm 4.7$ & $(21.5-42.8)$ \\
Carbohydrate & $\%$ energy & $58.1 \pm 7.4$ & $(33.3-75.9)$ & $58.4 \pm 7.3$ & $(33.3-75.9)$ & $53.7 \pm 6.7$ & $(38.2-65.3)$ \\
\hline
\end{tabular}

\subsection{Polyphenol Intake}

The polyphenol intake is summarized in Figure 1 and Table 2. The subjects consumed $1492 \pm 665 \mathrm{mg} /$ day of polyphenols on average (range 183-4854 mg/day). The average polyphenol intake from beverages was $1180 \pm 629 \mathrm{mg} /$ day, accounting for $79.1 \%$ of the total polyphenol intake, and the average polyphenol intake from food was $312 \pm 126 \mathrm{mg} /$ day, accounting for $20.9 \%$. The polyphenol intakes from non-alcoholic beverages and alcoholic beverages were $1104 \pm 607 \mathrm{mg} /$ day $(74.0 \%)$ and $76 \pm 166 \mathrm{mg} /$ day $(5.1 \%)$, respectively. Of the food groups, the portions of the polyphenol intake provided by vegetables was $6.8 \%$; that by cereals, $3.2 \%$; fruits, $3.0 \%$; beans, $2.9 \%$; and seasonings, $2.7 \%$. For both the men and women, approx. $80 \%$ of their polyphenol intake was from beverages and approx. $20 \%$ was from food.

The proportions and ranking of the polyphenol intake in this elderly Japanese population are shown in Table 3. Only small differences between men and women in the contribution of various food items was apparent. Although green tea was the most consumed beverage at $345 \pm 306 \mathrm{~mL} /$ day (approx. 2.3 cups) and coffee was the second largest one at $329 \pm 263 \mathrm{~mL} /$ day (approx. 2.2 cups), coffee was the largest source of polyphenol intake from all food and beverages, accounting for $43.2 \%$. Green tea, the second largest source, accounted for $26.6 \%$. The two major beverages together accounted for approx. 70\%, and beverages other than green tea and coffee accounted for at most 3\%. The third largest source of polyphenols in the men was beer at 3.0\% (in the women, beer ranked 30th at $0.3 \%$ ), and the third largest source of polyphenols in the women was black tea at $4.6 \%$ (in the men, black tea ranked 10 th at $1.4 \%$ ). 
Table 2. The subjects' polyphenol intake from beverages and foods.

\begin{tabular}{|c|c|c|c|c|c|c|c|c|c|}
\hline & \multicolumn{3}{|c|}{ Men and Women $(n=610)$} & \multicolumn{3}{|c|}{ Men $(n=569)$} & \multicolumn{3}{|c|}{ Women $(n=41)$} \\
\hline & \multicolumn{2}{|c|}{$\begin{array}{l}\text { Polyphenol Intake } \\
\text { (mg/Day) }\end{array}$} & \multirow{2}{*}{$\begin{array}{l}\text { Polyphenol } \\
\text { Intake (\%) }\end{array}$} & \multicolumn{2}{|c|}{$\begin{array}{l}\text { Polyphenol Intake } \\
\text { (mg/Day) }\end{array}$} & \multirow{2}{*}{$\begin{array}{l}\text { Polyphenol } \\
\text { Intake (\%) }\end{array}$} & \multicolumn{2}{|c|}{$\begin{array}{l}\text { Polyphenol Intake } \\
\text { (mg/Day) }\end{array}$} & \multirow{2}{*}{$\begin{array}{l}\text { Polyphenol } \\
\text { Intake (\%) }\end{array}$} \\
\hline & Mean \pm SD & Min-Max & & Mean \pm SD & Min-Max & & Mean \pm SD & Min-Max & \\
\hline Total & $1492 \pm 665$ & $(183-4854)$ & $100 \%$ & $1503 \pm 669$ & $(183-4854)$ & $100 \%$ & $1326 \pm 583$ & $(410-2970)$ & $100 \%$ \\
\hline Beverages & $1180 \pm 629$ & $(0-4380)$ & $79.1 \%$ & $1190 \pm 634$ & $(0-4380)$ & $79.1 \%$ & $1040 \pm 545$ & $(252-2497)$ & $78.4 \%$ \\
\hline Non alcoholic beverages & $1104 \pm 607$ & $(0-4058)$ & $74.0 \%$ & $1110 \pm 611$ & $(0-4058)$ & $73.8 \%$ & $1025 \pm 553$ & $(118-2497)$ & $77.3 \%$ \\
\hline Alcoholic beverages & $76 \pm 166$ & $(0-2307)$ & $5.1 \%$ & $80 \pm 171$ & $(0-2307)$ & $5.3 \%$ & $15 \pm 32$ & $(0-134)$ & $1.2 \%$ \\
\hline Foods & $312 \pm 126$ & $(71-1013)$ & $20.9 \%$ & $314 \pm 127$ & $(71-1013)$ & $20.9 \%$ & $286 \pm 101$ & $(114-543)$ & $21.6 \%$ \\
\hline Vegetables & $102 \pm 63$ & $(8-457)$ & $6.8 \%$ & $102 \pm 63$ & $(8-457)$ & $6.8 \%$ & $95 \pm 58$ & $(24-312)$ & $7.2 \%$ \\
\hline Cereals & $47 \pm 31$ & $(0-190)$ & $3.2 \%$ & $49 \pm 31$ & (0-190) & $3.2 \%$ & $30 \pm 15$ & $(6-79)$ & $2.3 \%$ \\
\hline Fruits & $44 \pm 35$ & $(0-209)$ & $3.0 \%$ & $44 \pm 35$ & (0-209) & $2.9 \%$ & $49 \pm 33$ & $(6-119)$ & $3.7 \%$ \\
\hline Pulses & $44 \pm 33$ & $(0-212)$ & $2.9 \%$ & $44 \pm 33$ & $(0-212)$ & $2.9 \%$ & $45 \pm 31$ & $(5-146)$ & $3.4 \%$ \\
\hline Seasonings & $40 \pm 13$ & $(12-104)$ & $2.7 \%$ & $41 \pm 13$ & $(12-104)$ & $2.7 \%$ & $31 \pm 13$ & $(12-72)$ & $2.4 \%$ \\
\hline Confectioneries & $14 \pm 27$ & $(0-390)$ & $0.9 \%$ & $14 \pm 27$ & $(0-390)$ & $0.9 \%$ & $13 \pm 15$ & $(0-61)$ & $1.0 \%$ \\
\hline Nuts and seeds & $14 \pm 14$ & $(0-116)$ & $0.9 \%$ & $14 \pm 14$ & $(0-116)$ & $0.9 \%$ & $17 \pm 14$ & $(0-53)$ & $1.3 \%$ \\
\hline Potatoes & $4 \pm 4$ & $(0-29)$ & $0.3 \%$ & $4 \pm 4$ & $(0-29)$ & $0.3 \%$ & $4 \pm 3$ & $(0-10)$ & $0.3 \%$ \\
\hline Algae & $2 \pm 2$ & $(0-15)$ & $0.1 \%$ & $2 \pm 2$ & $(0-15)$ & $0.1 \%$ & $2 \pm 2$ & $(0-5)$ & $0.1 \%$ \\
\hline
\end{tabular}




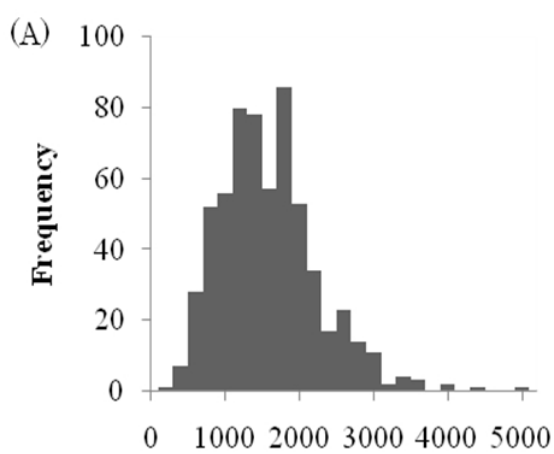

Polyphenolintake (mg/day)

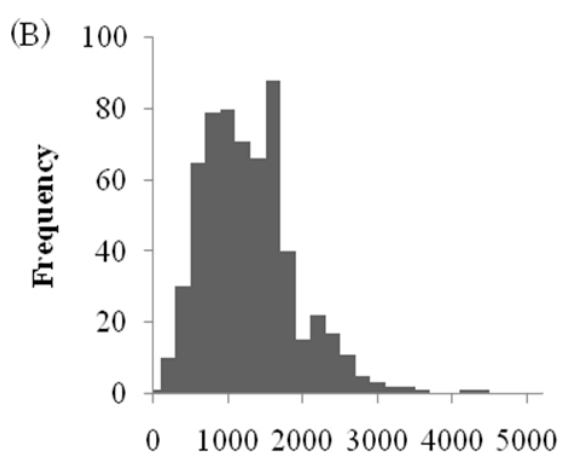

Polyphenol intake from beverage (mg/day)

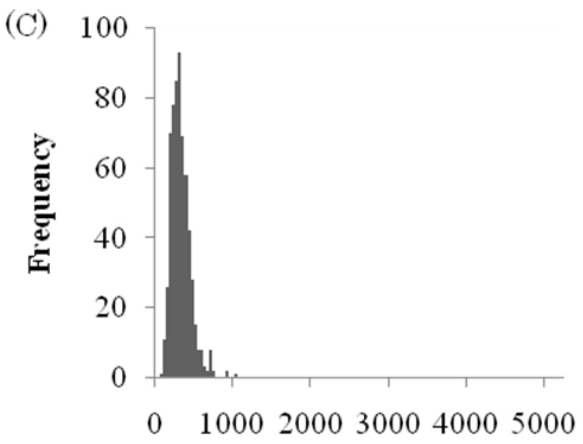

Polyphenol intake from food (mg/day)

Figure 1. Histograms of total polyphenol intake (A); polyphenol intake from beverages (B); and polyphenol intake from foods (C).

Table 3. The proportion and ranking of polyphenol intake in the subjects.

\begin{tabular}{|c|c|c|c|c|c|c|}
\hline & & \multirow[t]{2}{*}{ Type } & \multicolumn{2}{|c|}{$\begin{array}{l}\text { Polyphenol Intake } \\
\text { (mg/Day) }\end{array}$} & \multirow{2}{*}{$\begin{array}{c}\text { Polyphenol } \\
\text { Intake (\%) }\end{array}$} & \multirow{2}{*}{$\begin{array}{c}\text { Cumulative } \\
\text { Polyphenol } \\
\text { Intake (\%) }\end{array}$} \\
\hline & & & Mean \pm SD & Min-Max & & \\
\hline \multicolumn{7}{|c|}{ Men and women $(n=610)$} \\
\hline 1 & Coffee & B & $645 \pm 517$ & $(0-2613)$ & $43.2 \%$ & $43.2 \%$ \\
\hline 2 & Green tea & B & $397 \pm 351$ & $(0-1380)$ & $26.6 \%$ & $69.9 \%$ \\
\hline 3 & Beer & B & $43 \pm 63$ & $(0-513)$ & $2.9 \%$ & $72.7 \%$ \\
\hline 4 & Red wine & B & $33 \pm 152$ & $(0-2243)$ & $2.2 \%$ & $74.9 \%$ \\
\hline 5 & Tomato vegetable juice & V & $28 \pm 44$ & $(0-311)$ & $1.9 \%$ & $76.8 \%$ \\
\hline 6 & Spinach/broccoli & $\mathrm{V}$ & $26 \pm 18$ & $(0-107)$ & $1.7 \%$ & $78.5 \%$ \\
\hline 7 & Soy sauce & $S$ & $23 \pm 7$ & $(8-51)$ & $1.6 \%$ & $80.1 \%$ \\
\hline 8 & Black tea & B & $23 \pm 62$ & $(0-432)$ & $1.6 \%$ & $81.6 \%$ \\
\hline 9 & Buckwheat noodle & C & $22 \pm 26$ & $(0-162)$ & $1.5 \%$ & $83.1 \%$ \\
\hline 10 & Natto & $\mathrm{P}$ & $21 \pm 23$ & $(0-142)$ & $1.4 \%$ & $84.5 \%$ \\
\hline 11 & Tofu/fried tofu & $\mathrm{P}$ & $20 \pm 13$ & $(0-77)$ & $1.3 \%$ & $85.9 \%$ \\
\hline 12 & Oolong tea & B & $19 \pm 55$ & $(0-468)$ & $1.3 \%$ & $87.2 \%$ \\
\hline 13 & Onion/welsh onion & $\mathrm{V}$ & $17 \pm 12$ & $(0-70)$ & $1.1 \%$ & $88.3 \%$ \\
\hline 14 & Orange & $\mathrm{F}$ & $16 \pm 19$ & $(0-121)$ & $1.1 \%$ & $89.4 \%$ \\
\hline 15 & Miso & S & $14 \pm 10$ & $(0-61)$ & $1.0 \%$ & $90.4 \%$ \\
\hline 16 & Bread & $\mathrm{C}$ & $14 \pm 9$ & $(0-46)$ & $1.0 \%$ & $91.3 \%$ \\
\hline 17 & Chocolate & Co & $14 \pm 27$ & $(0-390)$ & $0.9 \%$ & $92.2 \%$ \\
\hline 18 & Barley tea & B & $13 \pm 23$ & $(0-108)$ & $0.9 \%$ & $93.1 \%$ \\
\hline 19 & Strawberry & $\mathrm{F}$ & $11 \pm 14$ & $(0-111)$ & $0.8 \%$ & $93.9 \%$ \\
\hline 20 & Nuts & $\mathrm{N}$ & $8 \pm 11$ & $(0-80)$ & $0.6 \%$ & $94.4 \%$ \\
\hline
\end{tabular}


Table 3. Cont.

\begin{tabular}{|c|c|c|c|c|c|c|}
\hline & & \multirow[t]{2}{*}{ Type } & \multicolumn{2}{|c|}{$\begin{array}{l}\text { Polyphenol Intake } \\
\text { (mg/Day) }\end{array}$} & \multirow{2}{*}{$\begin{array}{l}\text { Polyphenol } \\
\text { Intake (\%) }\end{array}$} & \multirow{2}{*}{$\begin{array}{l}\text { Cumulative } \\
\text { Polyphenol } \\
\text { Intake (\%) }\end{array}$} \\
\hline & & & Mean \pm SD & Min-Max & & \\
\hline \multicolumn{7}{|c|}{ Men and women $(n=610)$} \\
\hline 21 & Egg plant & V & $8 \pm 7$ & $(0-55)$ & $0.5 \%$ & $95.0 \%$ \\
\hline 22 & Fruit juice & $\mathrm{F}$ & $7 \pm 12$ & $(0-102)$ & $0.5 \%$ & $95.4 \%$ \\
\hline 23 & Apple/banana & $\mathrm{F}$ & $7 \pm 5$ & $(0-25)$ & $0.5 \%$ & $95.9 \%$ \\
\hline 24 & Green soybean & $\mathrm{V}$ & $6 \pm 7$ & $(0-52)$ & $0.4 \%$ & $96.3 \%$ \\
\hline 25 & Sesame & $\mathrm{N}$ & $6 \pm 7$ & $(0-54)$ & $0.4 \%$ & $96.7 \%$ \\
\hline 26 & Cocoa/chocolate drink & $\mathrm{B}$ & $5 \pm 21$ & $(0-186)$ & $0.3 \%$ & $97.0 \%$ \\
\hline 27 & Potato & Po & $4 \pm 4$ & $(0-29)$ & $0.3 \%$ & $97.3 \%$ \\
\hline 28 & Cabbage & $\mathrm{V}$ & $4 \pm 2$ & $(0-15)$ & $0.2 \%$ & $97.5 \%$ \\
\hline 29 & Soy milk & $\mathrm{P}$ & $3 \pm 14$ & $(0-162)$ & $0.2 \%$ & $97.7 \%$ \\
\hline 30 & Udon noodle & $\mathrm{C}$ & $3 \pm 3$ & $(0-21)$ & $0.2 \%$ & $98.0 \%$ \\
\hline 31 & Japanese radish/turnip & $\mathrm{V}$ & $3 \pm 3$ & $(0-23)$ & $0.2 \%$ & $98.2 \%$ \\
\hline 32 & Prune & $\mathrm{F}$ & $3 \pm 12$ & $(0-72)$ & $0.2 \%$ & $98.4 \%$ \\
\hline 33 & Parsley/perilla & $\mathrm{V}$ & $3 \pm 4$ & $(0-31)$ & $0.2 \%$ & $98.6 \%$ \\
\hline 34 & Chinese noodle & $\mathrm{C}$ & $3 \pm 3$ & $(0-22)$ & $0.2 \%$ & $98.7 \%$ \\
\hline 35 & Curry powder & $\mathrm{S}$ & $2 \pm 2$ & $(0-18)$ & $0.2 \%$ & $98.9 \%$ \\
\hline 36 & Ginger & $\mathrm{V}$ & $2 \pm 2$ & $(0-24)$ & $0.2 \%$ & $99.1 \%$ \\
\hline 37 & Rice & $\mathrm{C}$ & $2 \pm 1$ & $(0-7)$ & $0.1 \%$ & $99.2 \%$ \\
\hline 38 & Pasta & $\mathrm{C}$ & $2 \pm 2$ & $(0-24)$ & $0.1 \%$ & $99.3 \%$ \\
\hline 39 & Other teas & $\mathrm{B}$ & $2 \pm 8$ & $(0-54)$ & $0.1 \%$ & $99.5 \%$ \\
\hline 40 & Laver & A & $2 \pm 2$ & $(0-15)$ & $0.1 \%$ & $99.6 \%$ \\
\hline \multicolumn{7}{|c|}{$\operatorname{Men}(n=569)$} \\
\hline 1 & Coffee & B & $652 \pm 520$ & $(0-2613)$ & $43.4 \%$ & $43.4 \%$ \\
\hline 2 & Green tea & $\mathrm{B}$ & $400 \pm 354$ & $(0-1380)$ & $26.6 \%$ & $69.9 \%$ \\
\hline 3 & Beer & B & $46 \pm 64$ & $(0-513)$ & $3.0 \%$ & $73.0 \%$ \\
\hline 4 & Red wine & $\mathrm{B}$ & $34 \pm 157$ & $(0-2243)$ & $2.3 \%$ & $75.3 \%$ \\
\hline 5 & Tomato vegetable juice & $\mathrm{V}$ & $29 \pm 45$ & $(0-311)$ & $1.9 \%$ & $77.2 \%$ \\
\hline 6 & Spinach/broccoli & $\mathrm{V}$ & $26 \pm 18$ & $(0-107)$ & $1.7 \%$ & $78.9 \%$ \\
\hline 7 & Soy sauce & S & $24 \pm 7$ & $(10-51)$ & $1.6 \%$ & $80.4 \%$ \\
\hline 8 & Buckwheat noodle & $\mathrm{C}$ & $23 \pm 27$ & $(0-162)$ & $1.6 \%$ & $82.0 \%$ \\
\hline 9 & Natto & $\mathrm{P}$ & $21 \pm 23$ & $(0-142)$ & $1.4 \%$ & $83.4 \%$ \\
\hline 10 & Black tea & $\mathrm{B}$ & $20 \pm 56$ & $(0-432)$ & $1.4 \%$ & $84.7 \%$ \\
\hline 11 & Tofu/fried tofu & $\mathrm{P}$ & $20 \pm 13$ & $(0-75)$ & $1.3 \%$ & $86.1 \%$ \\
\hline 12 & Oolong tea & B & $19 \pm 54$ & $(0-468)$ & $1.3 \%$ & $87.3 \%$ \\
\hline 13 & Onion/welsh onion & $\mathrm{V}$ & $17 \pm 12$ & $(0-70)$ & $1.1 \%$ & $88.5 \%$ \\
\hline 14 & Orange & $\mathrm{F}$ & $16 \pm 19$ & $(0-121)$ & $1.1 \%$ & $89.6 \%$ \\
\hline 15 & Miso & $S$ & $15 \pm 10$ & $(0-61)$ & $1.0 \%$ & $90.5 \%$ \\
\hline 16 & Bread & $\mathrm{C}$ & $14 \pm 9$ & $(0-46)$ & $1.0 \%$ & $91.5 \%$ \\
\hline 17 & Chocolate & Co & $14 \pm 27$ & $(0-390)$ & $0.9 \%$ & $92.4 \%$ \\
\hline 18 & Barley tea & $\mathrm{B}$ & $13 \pm 23$ & $(0-108)$ & $0.9 \%$ & $93.3 \%$ \\
\hline 19 & Strawberry & $\mathrm{F}$ & $11 \pm 14$ & $(0-111)$ & $0.7 \%$ & $94.0 \%$ \\
\hline 20 & Nuts & $\mathrm{N}$ & $8 \pm 11$ & $(0-80)$ & $0.5 \%$ & $94.5 \%$ \\
\hline 21 & Egg plant & $\mathrm{V}$ & $8 \pm 7$ & $(0-55)$ & $0.5 \%$ & $95.1 \%$ \\
\hline 22 & Fruit juice & $\mathrm{F}$ & $7 \pm 12$ & $(0-102)$ & $0.5 \%$ & $95.5 \%$ \\
\hline 23 & Apple/banana & $\mathrm{F}$ & $7 \pm 5$ & $(0-25)$ & $0.5 \%$ & $95.9 \%$ \\
\hline 24 & Green soybean & $\mathrm{V}$ & $6 \pm 7$ & $(0-52)$ & $0.4 \%$ & $96.4 \%$ \\
\hline 25 & Sesame & $\mathrm{N}$ & $5 \pm 6$ & $(0-54)$ & $0.4 \%$ & $96.7 \%$ \\
\hline 26 & Cabbage & V & $4 \pm 4$ & $(0-29)$ & $0.3 \%$ & $97.0 \%$ \\
\hline 27 & Cocoa/chocolate drink & $\mathrm{B}$ & $4 \pm 19$ & $(0-186)$ & $0.3 \%$ & $97.3 \%$ \\
\hline 28 & Potato & Po & $4 \pm 2$ & $(0-15)$ & $0.2 \%$ & $97.5 \%$ \\
\hline 29 & Udon noodle & $\mathrm{C}$ & $3 \pm 3$ & $(0-21)$ & $0.2 \%$ & $97.8 \%$ \\
\hline 30 & Japanese radish/turnip & $\mathrm{V}$ & $3 \pm 3$ & $(0-23)$ & $0.2 \%$ & $98.0 \%$ \\
\hline 31 & Soy milk & $\mathrm{P}$ & $3 \pm 14$ & $(0-162)$ & $0.2 \%$ & $98.2 \%$ \\
\hline 32 & Prune & $\mathrm{F}$ & $3 \pm 12$ & $(0-72)$ & $0.2 \%$ & $98.4 \%$ \\
\hline
\end{tabular}


Table 3. Cont.

\begin{tabular}{|c|c|c|c|c|c|c|}
\hline & & \multirow[t]{2}{*}{ Type } & \multicolumn{2}{|c|}{$\begin{array}{l}\text { Polyphenol Intake } \\
\text { (mg/Day) }\end{array}$} & \multirow{2}{*}{$\begin{array}{c}\text { Polyphenol } \\
\text { Intake (\%) }\end{array}$} & \multirow{2}{*}{$\begin{array}{l}\text { Cumulative } \\
\text { Polyphenol } \\
\text { Intake (\%) }\end{array}$} \\
\hline & & & Mean \pm SD & Min-Max & & \\
\hline \multicolumn{7}{|c|}{ Men $(n=569)$} \\
\hline 33 & Chinese noodle & $\mathrm{C}$ & $3 \pm 3$ & $(0-22)$ & $0.2 \%$ & $98.6 \%$ \\
\hline 34 & Parsley/perilla & $\mathrm{V}$ & $3 \pm 4$ & $(0-31)$ & $0.2 \%$ & $98.8 \%$ \\
\hline 35 & Curry powder & $S$ & $2 \pm 2$ & $(0-18)$ & $0.2 \%$ & $98.9 \%$ \\
\hline 36 & Ginger & $\mathrm{V}$ & $2 \pm 2$ & $(0-24)$ & $0.2 \%$ & $99.1 \%$ \\
\hline 37 & Rice & $\mathrm{C}$ & $2 \pm 1$ & $(0-7)$ & $0.2 \%$ & $99.2 \%$ \\
\hline 38 & Pasta & $\mathrm{C}$ & $2 \pm 2$ & $(0-24)$ & $0.1 \%$ & $99.4 \%$ \\
\hline 39 & Laver & $\mathrm{A}$ & $2 \pm 2$ & $(0-15)$ & $0.1 \%$ & $99.5 \%$ \\
\hline 40 & Other teas & $\mathrm{B}$ & $2 \pm 7$ & $(0-54)$ & $0.1 \%$ & $99.6 \%$ \\
\hline \multicolumn{7}{|c|}{ Women $(n=41)$} \\
\hline 1 & Coffee & B & $549 \pm 464$ & $(0-2250)$ & $41.4 \%$ & $41.4 \%$ \\
\hline 2 & Green tea & B & $359 \pm 319$ & (0-1380) & $27.1 \%$ & $68.4 \%$ \\
\hline 3 & Black tea & B & $61 \pm 112$ & $(0-432)$ & $4.6 \%$ & $73.0 \%$ \\
\hline 4 & Spinach/broccoli & $\mathrm{V}$ & $25 \pm 18$ & $(3-85)$ & $1.9 \%$ & $75.0 \%$ \\
\hline 5 & Tomato vegetable juice & $\mathrm{V}$ & $23 \pm 38$ & $(0-104)$ & $1.7 \%$ & $76.7 \%$ \\
\hline 6 & Tofu/fried tofu & $\mathrm{P}$ & $22 \pm 13$ & $(2-77)$ & $1.7 \%$ & $78.3 \%$ \\
\hline 7 & Oolong tea & B & $21 \pm 63$ & $(0-263)$ & $1.6 \%$ & $79.9 \%$ \\
\hline 8 & Barley tea & B & $21 \pm 27$ & $(0-88)$ & $1.6 \%$ & $81.5 \%$ \\
\hline 9 & Orange & $\mathrm{F}$ & $17 \pm 16$ & $(0-53)$ & $1.3 \%$ & $82.7 \%$ \\
\hline 10 & Soy sauce & $S$ & $17 \pm 5$ & $(8-28)$ & $1.3 \%$ & $84.0 \%$ \\
\hline 11 & Natto & $\mathrm{P}$ & $16 \pm 19$ & $(0-62)$ & $1.2 \%$ & $85.2 \%$ \\
\hline 12 & Onion/welsh onion & $\mathrm{V}$ & $14 \pm 11$ & $(2-61)$ & $1.1 \%$ & $86.3 \%$ \\
\hline 13 & Bread & $\mathrm{C}$ & $13 \pm 7$ & $(0-32)$ & $1.0 \%$ & $87.3 \%$ \\
\hline 14 & Miso & $S$ & $13 \pm 11$ & $(0-53)$ & $1.0 \%$ & $88.3 \%$ \\
\hline 15 & Chocolate & Co & $13 \pm 15$ & $(0-61)$ & $1.0 \%$ & $89.2 \%$ \\
\hline 16 & Red wine & $\mathrm{B}$ & $12 \pm 29$ & $(0-123)$ & $0.9 \%$ & $90.2 \%$ \\
\hline 17 & Strawberry & $\mathrm{F}$ & $12 \pm 13$ & $(1-54)$ & $0.9 \%$ & $91.0 \%$ \\
\hline 18 & Egg plant & $\mathrm{V}$ & $11 \pm 12$ & $(0-55)$ & $0.8 \%$ & $91.8 \%$ \\
\hline 19 & Buckwheat noodle & $\mathrm{C}$ & $10 \pm 12$ & $(0-50)$ & $0.8 \%$ & $92.6 \%$ \\
\hline 20 & Nuts & $\mathrm{N}$ & $9 \pm 11$ & $(0-40)$ & $0.7 \%$ & $93.3 \%$ \\
\hline 21 & Fruit juice & $\mathrm{F}$ & $9 \pm 16$ & $(0-51)$ & $0.7 \%$ & $94.0 \%$ \\
\hline 22 & Cocoa/chocolate drink & $\mathrm{B}$ & $9 \pm 33$ & $(0-186)$ & $0.7 \%$ & $94.7 \%$ \\
\hline 23 & Apple/banana & $\mathrm{F}$ & $8 \pm 5$ & $(1-22)$ & $0.6 \%$ & $95.3 \%$ \\
\hline 24 & Sesame & $\mathrm{N}$ & $8 \pm 8$ & $(0-36)$ & $0.6 \%$ & $95.9 \%$ \\
\hline 25 & Soy milk & $\mathrm{P}$ & $6 \pm 15$ & $(0-54)$ & $0.5 \%$ & $96.4 \%$ \\
\hline 26 & Other teas & B & $6 \pm 12$ & $(0-36)$ & $0.4 \%$ & $96.8 \%$ \\
\hline 27 & Green soybean & $\mathrm{V}$ & $5 \pm 5$ & $(0-17)$ & $0.4 \%$ & $97.2 \%$ \\
\hline 28 & Cabbage & $\mathrm{V}$ & $4 \pm 3$ & $(0-10)$ & $0.3 \%$ & $97.5 \%$ \\
\hline 29 & Parsley/perilla & $\mathrm{V}$ & $4 \pm 6$ & $(0-31)$ & $0.3 \%$ & $97.8 \%$ \\
\hline 30 & Beer & $\mathrm{B}$ & $3 \pm 9$ & $(0-46)$ & $0.3 \%$ & $98.1 \%$ \\
\hline 31 & Potato & $\mathrm{Po}$ & $3 \pm 2$ & $(0-11)$ & $0.2 \%$ & $98.3 \%$ \\
\hline 32 & Ginger & $\mathrm{V}$ & $3 \pm 2$ & $(0-8)$ & $0.2 \%$ & $98.5 \%$ \\
\hline 33 & Japanese radish/turnip & $\mathrm{V}$ & $2 \pm 2$ & $(0-10)$ & $0.2 \%$ & $98.7 \%$ \\
\hline 34 & Prune & $\mathrm{F}$ & $2 \pm 11$ & $(0-72)$ & $0.2 \%$ & $98.8 \%$ \\
\hline 35 & Curry powder & $\mathrm{S}$ & $2 \pm 1$ & $(0-3)$ & $0.2 \%$ & $99.0 \%$ \\
\hline 36 & Udon noodle & $\mathrm{C}$ & $2 \pm 2$ & $(0-9)$ & $0.1 \%$ & $99.1 \%$ \\
\hline 37 & Laver & $\mathrm{A}$ & $2 \pm 2$ & $(0-5)$ & $0.1 \%$ & $99.3 \%$ \\
\hline 38 & Pasta & $\mathrm{C}$ & $2 \pm 2$ & $(0-15)$ & $0.1 \%$ & $99.4 \%$ \\
\hline 39 & Rice & $\mathrm{C}$ & $2 \pm 1$ & $(0-4)$ & $0.1 \%$ & $99.5 \%$ \\
\hline 40 & Chinese noodle & $\mathrm{C}$ & $1 \pm 1$ & $(0-7)$ & $0.1 \%$ & $99.6 \%$ \\
\hline
\end{tabular}

B, beverages; V, vegetables; $\mathrm{S}$, seasonings; $\mathrm{C}$, cereals; $\mathrm{P}$, pulses; F, fruits; Co, confectioneries; N, Nuts and seeds; Po, Potatoes; A, Algae.

The top 10 items (composed of four food items and six beverages) and the top 20 items (12 food and eight beverages) accounted for more than $80 \%$ and $90 \%$ of the cumulative daily consumption of 
polyphenols, respectively. As for food, although its contribution was small, spinach/broccoli $(1.7 \%)$, soy sauce $(1.6 \%)$, buckwheat noodles $(1.5 \%)$, natto (i.e., fermented soybeans) $(1.4 \%)$, tofu (i.e., soybean curd)/fried tofu (1.3\%), onion/welsh onion (1.1\%), miso (i.e., fermented soybean paste) $(0.97 \%)$, bread $(0.96 \%)$ and chocolate $(0.93 \%)$ contributed to the polyphenol intake. Soybean products such as soy sauce, natto, tofu/fried tofu, miso, and soy milk together accounted for $5.4 \%$, as the third largest contributor after coffee and green tea.

\subsection{Association between Polyphenol Intake and Micronutrient Intake}

The correlation coefficients of polyphenol intake with energy-adjusted micronutrient intakes are shown in Table 4 . We calculated the energy-adjusted values of micronutrient intake by the density method. We found that the polyphenol intake was not strongly associated with any of the micronutrients. Highest correlations were found for niacin, magnesium and potassium $(r=0.266$, 0.245 and 0.223 , respectively).

Table 4. Correlation coefficients of energy-adjusted micronutrient intakes with polyphenol intake.

\begin{tabular}{|c|c|}
\hline Nutrients & $r$ \\
\hline Energy & 0.317 \\
\hline Niacin * & 0.266 \\
\hline Magnesium * & 0.245 \\
\hline Potassium * & 0.223 \\
\hline Manganese * & 0.187 \\
\hline Folate * & 0.182 \\
\hline Vitamin $C^{*}$ & 0.180 \\
\hline Iron * & 0.156 \\
\hline Riboflavin * & 0.150 \\
\hline Copper* & 0.126 \\
\hline Fiber * & 0.123 \\
\hline$\beta$-Carotene equivalent * & 0.118 \\
\hline Vitamin $K^{*}$ & 0.117 \\
\hline Phosphorus * & 0.117 \\
\hline$\alpha$-Tocopherol * & 0.107 \\
\hline Vitamin B6 * & 0.106 \\
\hline Vitamin B12 * & 0.106 \\
\hline Pantothenic acid * & 0.104 \\
\hline Vitamin A (retinol equivalent) * & 0.097 \\
\hline Calcium* & 0.087 \\
\hline Thiamin * & 0.085 \\
\hline Zinc * & 0.078 \\
\hline Vitamin D* & 0.060 \\
\hline Retinol* & 0.050 \\
\hline Sodium * & 0.012 \\
\hline
\end{tabular}

Values are the Spearman's correlation coefficients. ${ }^{*}$ Energy-adjusted micronutrient intakes by the density method.

\section{Discussion}

Our present findings revealed that in a population of elderly Japanese, mostly (93\%) men, consumed on average $1492 \pm 665 \mathrm{mg} /$ person/day of polyphenol $(1503 \pm 669 \mathrm{mg} /$ day in the men and $1326 \pm 583 \mathrm{mg} /$ day in the women). The dietary polyphenol intake was largely composed of beverages, which accounted for $79 \%$ of the intake. Coffee was the largest source of polyphenol intake at $43 \%$, followed by green tea at $27 \%$. This is in line with our previous study showing that beverages accounted for approx. $80 \%$ of the total intake, and that coffee and green tea were the major sources of dietary polyphenol [30].

Compared to our earlier study, which demonstrated that middle-aged Japanese women consumed $841 \pm 406 \mathrm{mg} /$ day of polyphenol [30], the elderly Japanese in the present study consumed 
a greater amount of polyphenol. Our recent study using a beverage survey of approx. 10,000 Japanese men and women indicated that the polyphenol intake from beverages was larger in the elderly than in the young or middle-aged and greater among the men than the women, and that the polyphenol intake was influenced by the amounts of coffee and green tea consumed [30]. The elderly women in this study consumed larger amounts of polyphenol compared to the middle-aged women in our previous study. Therefore, polyphenol intake of elderly Japanese might be higher than that of Japanese adult for both men and women.

It is also noteworthy that the daily polyphenol consumption varied considerably among the individuals, ranging from 183 to $4854 \mathrm{mg}$ /day (Figure 1). The polyphenol intake from food showed less individual variation compared to the polyphenol intake from beverages. Coffee and green tea consumption had large impacts on the subjects' polyphenol intake, and the amount of coffee and green tea consumption varied according to individual preference. Our studies suggest that the amount of beverage consumption, especially coffee and green tea, is a principal factor in the individual variation of polyphenol intake among the Japanese.

As shown in Table 3, the top 10 food and beverage items accounted for $>80 \%$ of the total polyphenol intake, and the top 20 items accounted for $>90 \%$, indicating that a very limited variety of items could contribute to the dietary polyphenol intake by elderly Japanese. Seventeen of the top 20 food and beverage items identified in the present study were in common with those identified in middle-aged women in our previous study (i.e., coffee, green tea, beer, red wine, tomato vegetable juice, soy sauce, black tea, buckwheat noodle, natto, tofu, oolong tea, onion, orange, miso, bread, chocolate, and barley tea), although the ranking order was partly different: the rankings of beer and red wine were higher in the elderly subjects. We found that the daily polyphenol intake in the subjects could be estimated by the assessment of about 10-20 food and beverage items with an accuracy rate at $80 \%-90 \%$. Our original database containing the polyphenol contents of 67 items covered the major contributors to polyphenol intake in Japan.

Several studies have reported daily polyphenol consumption in European countries, showing that it was $863 \pm 415 \mathrm{mg} /$ day in Finnish [38], $1193 \pm 510 \mathrm{mg} /$ day in French [10], $820 \pm 323 \mathrm{mg} /$ day in Spanish [39], and $1756.5 \pm 695.8 \mathrm{mg} /$ day in Polish [40], which have used the Phenol-Explorer database or similar quantitative value measured by HPLC methods. In order to compare our data to those in Europe, we calculated total polyphenol intake in this study's subjects using the Phenol-Explorer database. The total polyphenol intake was estimated to be $961 \pm 452 \mathrm{mg} /$ day, although 19 food and beverage items unlisted in the database could not be included for the calculation. The estimated value was remarkably lower than the value obtained by our database. The discrepancy might be due to the fact that the value for the total poplyphenol in coffee, the largest source of pholyphenols, is lower in Phenol-Explorer $(123.6 \mathrm{mg} / 100 \mathrm{~g}$, as calculated the usage of the two major cultivar Arabica and Robusta coffee beverage at $70 \%$ and 30\%, respectively, based on Japanese statics of import) than our database $(200 \mathrm{mg} / 100 \mathrm{~g})$. Coffee contains a mixture of coffee polyphenols including 5-caffeoyl quinic acid (5-CQA), other chlorogenic acid groups, and other phenolic compounds generated through roasting process. In the HPLC methods, it is difficult to measure all polyphenol molecules due to the limited standard samples, which causes to underestimate total polyphenol contents. In our unpublished data, 5-CQA and the other simple 7 chlorogenic acids made up $10 \%$ and $20 \%$ of total polyphenol, respectively, in the soluble coffee sample. On the contrary, the Folin-Ciocalteu method has advantages to roughly estimate overall amounts of polyphenols. Additionally, the lack of some Japanese food items, such as miso, buckwheat noodle and sweet bean paste in Phenol-Explorer might be another cause for the discrepancy. On the other hand, total polyphenol contents of some foods such as potato, apple and grape in the Phenol-Explorer database are much higher than that in our database. Japanese typically peel the skin of potato and fruits off and do not consume it. Such eating manner of food and/or difference in harvest or breeding manner should be different among countries and societies. Our original 
database for polyphenol contents is valuable to properly estimate total dietary polyphenol intakes in Japanese populations.

In this study, the elderly Japanese subjects consumed polyphenols largely from beverages, and less from vegetables, cereals, and fruits, while they were the major contributors to polyphenol intake in the other European countries. For example, French adults consumed a certain amount of polyphenols from fruits $(17 \%)$, vegetables $(7 \%)$ and cereals $(4 \%)$. On the other hand, soybeans play an important part in the polyphenol intake in the Japanese diet. It was reported that soy consumptions and isoflavone intakes were high in the Japanese population compared to those of other countries [41,42]. Food items made from soybeans such as tofu and natto and seasonings made from soybeans (i.e., soy sauce and miso) accounted for $5.4 \%$ of the total polyphenol intake in the present study.

As mentioned above, accumulating evidence indicates that polyphenol intake may contribute to reducing the risk of the development of several diseases and their related mortality rates [14-26]. However, it is still unclear whether these benefits for human health are due to the intakes of polyphenols and/or to the accompanying nutrients. In the present study, we also evaluated the relationship between polyphenol intake and nutritional intake, and interestingly, we found that the subjects' polyphenol intake was not associated strongly with the intake of any micronutrients, but it was very weakly associated with the intake of some micronutrients (i.e., niacin, magnesium and potassium). These findings may have been obtained because the Japanese are largely dependent on coffee and green tea for polyphenol consumption. In fact, weak but statistically significant associations between total polyphenol intake and some micronutrients such as magnesium, vitamin B6 and vitamin K were detected when these two beverages were excluded from the analysis $(r=0.291$, 0.289 and 0.280 , respectively). In countries such as Spain and France, the consumption of dietary polyphenols could be accompanied by that of specific nutrients derived from the consumption of fruits and/or vegetables. In future epidemiological studies, it will be necessary to determine the links between dietary polyphenol and nutrients in order to clarify the health benefits of a polyphenol-rich diet.

Some limitations of our study should be mentioned. First, the subjects in this study were all recruited from among the retired employees of a food manufacturing company, which may have caused a bias in the subject population who drank greater amounts of coffee (approx. $+100 \mathrm{~mL} /$ day) compared to the same age-group we observed in our previous study [31]. Although this study was not population-based and showed slightly higher coffee consumption, the observation we found in our series of studies showing that coffee and green tea are the largest sources of polyphenols in the Japanese diet remains consistent. Moreover, we used the original FFQ and polyphenol content database for estimating dietary polyphenol intake. In our unpublished data, total polyphenol intake estimated by the FFQs was significantly correlated with those obtained by the dietary records. Our database consists of a limited number of foods, but its weight-based coverage of the consumption of vegetable and fruit items with polyphenol content information was 94\%-95\% [30]. Finally, the number of women in the present study was very small at $6.7 \%$.

\section{Conclusions}

In conclusion, the present study showed that a population of elderly Japanese (mostly men) consumed $1492 \pm 665 \mathrm{mg}$ /day of polyphenols on average, which is slightly higher than previous data in Japanese adults, and coffee and green tea were the largest sources of polyphenols in their daily life. Our data will be valuable as additional baseline data of polyphenol intake in the Japanese and for future research focused on the relationship between polyphenols and their health benefits for humans.

Acknowledgments: We are grateful to the volunteers for participating in the study.

Author Contributions: Chie Taguchi, Yoichi Fukushima, Yoshimi Kishimoto and Kazuo Kondo contributed the design of the study. Chie Taguchi, Yoichi Fukushima and Yoshinari Takahashi conducted the data collection and 
analysis. Chie Taguchi and Norie Suzuki-Sugihara participated in the maintenance of the polyphenol database with assistance from Yoshimi Kishimoto and Emi Saita, Chie Taguchi, Yoichi Fukushima and Yoshimi Kishimoto prepared the manuscript. Kazuo Kondo supervised the study and reviewed the manuscript. All authors have read and approved the final manuscript.

Conflicts of Interest: The authors declare no conflict of interest.

\section{References}

1. Manach, C.; Scalbert, A.; Morand, C.; Remesy, C.; Jimenez, L. Polyphenols: Food sources and bioavailability. Am. J. Clin. Nutr. 2004, 79, 727-747. [PubMed]

2. Crozier, A.; Jaganath, I.B.; Clifford, M.N. Dietary phenolics: Chemistry, bioavailability and effects on health. Nat. Prod. Rep. 2009, 26, 1001-1043. [CrossRef] [PubMed]

3. Hertog, M.G.; Kromhout, D.; Aravanis, C.; Blackburn, H.; Buzina, R.; Fidanza, F.; Giampaoli, S.; Jansen, A.; Menotti, A.; Nedeljkovic, S.; et al. Flavonoid intake and long-term risk of coronary heart disease and cancer in the seven countries study. Arch. Intern. Med. 1995, 155, 381-386. [CrossRef] [PubMed]

4. Hertog, M.G.; Feskens, E.J.; Kromhout, D. Antioxidant flavonols and coronary heart disease risk. Lancet 1997, 349, 699. [CrossRef]

5. Landete, J.M. Dietary intake of natural antioxidants: Vitamins and polyphenols. Crit. Rev. Food Sci. Nutr. 2013, 53, 706-721. [CrossRef] [PubMed]

6. Del Rio, D.; Rodriguez-Mateos, A.; Spencer, J.P.; Tognolini, M.; Borges, G.; Crozier, A. Dietary (poly)phenolics in human health: Structures, bioavailability, and evidence of protective effects against chronic diseases. Antioxid. Redox Signal. 2013, 18, 1818-1892. [CrossRef] [PubMed]

7. USDA Nutrient Data Laboratory. Database for the Flavonoid Content of Selected Foods (Release 3.1. May 2014). Available online: http://www.ars.usda.gov/SP2UserFiles/Place/80400525/Data/Flav/ Flav_R03-1.pdf (accessed on 5 September 2015).

8. Neveu, V.; Perez-Jimenez, J.; Vos, F.; Crespy, V.; du Chaffaut, L.; Mennen, L.; Knox, C.; Eisner, R.; Cruz, J.; Wishart, D.; et al. Phenol-explorer: An online comprehensive database on polyphenol contents in foods. Database (Oxford) 2010. [CrossRef] [PubMed]

9. Rothwell, J.A.; Urpi-Sarda, M.; Boto-Ordonez, M.; Knox, C.; Llorach, R.; Eisner, R.; Cruz, J.; Neveu, V.; Wishart, D.; Manach, C.; et al. Phenol-explorer 2.0: A major update of the phenol-explorer database integrating data on polyphenol metabolism and pharmacokinetics in humans and experimental animals. Database (Oxford) 2012, 2012. [CrossRef] [PubMed]

10. Perez-Jimenez, J.; Fezeu, L.; Touvier, M.; Arnault, N.; Manach, C.; Hercberg, S.; Galan, P.; Scalbert, A. Dietary intake of 337 polyphenols in French adults. Am. J. Clin. Nutr. 2011, 93, 1220-1228. [CrossRef] [PubMed]

11. Zujko, M.E.; Witkowska, A.M.; Waskiewicz, A.; Sygnowska, E. Estimation of dietary intake and patterns of polyphenol consumption in Polish adult population. Adv. Med. Sci. 2012, 57, 375-384. [CrossRef] [PubMed]

12. Chun, O.K.; Chung, S.J.; Song, W.O. Estimated dietary flavonoid intake and major food sources of U.S. Adults. J. Nutr. 2007, 137, 1244-1252. [PubMed]

13. Zamora-Ros, R.; Knaze, V.; Rothwell, J.A.; Hemon, B.; Moskal, A.; Overvad, K.; Tjonneland, A.; Kyro, C.; Fagherazzi, G.; Boutron-Ruault, M.C.; et al. Dietary polyphenol intake in Europe: The European Prospective Investigation into Cancer and Nutrition (EPIC) study. Eur. J. Nutr. 2015, in print. [CrossRef] [PubMed]

14. Sesso, H.D.; Gaziano, J.M.; Liu, S.; Buring, J.E. Flavonoid intake and the risk of cardiovascular disease in women. Am. J. Clin. Nutr. 2003, 77, 1400-1408. [PubMed]

15. Mink, P.J.; Scrafford, C.G.; Barraj, L.M.; Harnack, L.; Hong, C.P.; Nettleton, J.A.; Jacobs, D.R., Jr. Flavonoid intake and cardiovascular disease mortality: A prospective study in postmenopausal women. Am. J. Clin. Nutr. 2007, 85, 895-909. [PubMed]

16. Mursu, J.; Voutilainen, S.; Nurmi, T.; Tuomainen, T.P.; Kurl, S.; Salonen, J.T. Flavonoid intake and the risk of ischaemic stroke and CVD mortality in middle-aged Finnish men: The Kuopio Ischaemic Heart Disease Risk Factor Study. Br. J. Nutr. 2008, 100, 890-895. [CrossRef] [PubMed]

17. Tresserra-Rimbau, A.; Rimm, E.B.; Medina-Remon, A.; Martinez-Gonzalez, M.A.; de la Torre, R.; Corella, D.; Salas-Salvado, J.; Gomez-Gracia, E.; Lapetra, J.; Aros, F.; et al. Inverse association between habitual polyphenol intake and incidence of cardiovascular events in the PREDIMED study. Nutr. Metab. Cardiovasc. Dis. 2014, 24, 639-647. [CrossRef] [PubMed] 
18. McCullough, M.L.; Peterson, J.J.; Patel, R.; Jacques, P.F.; Shah, R.; Dwyer, J.T. Flavonoid intake and cardiovascular disease mortality in a prospective cohort of US adults. Am. J. Clin. Nutr. 2012, 95, 454-464. [CrossRef] [PubMed]

19. Van Dam, R.M.; Naidoo, N.; Landberg, R. Dietary flavonoids and the development of type 2 diabetes and cardiovascular diseases: Review of recent findings. Curr. Opin. Lipidol. 2013, 24, 25-33. [CrossRef] [PubMed]

20. Zamora-Ros, R.; Touillaud, M.; Rothwell, J.A.; Romieu, I.; Scalbert, A. Measuring exposure to the polyphenol metabolome in observational epidemiologic studies: Current tools and applications and their limits. Am. J. Clin. Nutr. 2014, 100, 11-26. [CrossRef] [PubMed]

21. Cutler, G.J.; Nettleton, J.A.; Ross, J.A.; Harnack, L.J.; Jacobs, D.R., Jr.; Scrafford, C.G.; Barraj, L.M.; Mink, P.J.; Robien, K. Dietary flavonoid intake and risk of cancer in postmenopausal women: The iowa women's health study. Int. J. Cancer 2008, 123, 664-671. [CrossRef] [PubMed]

22. Bobe, G.; Peterson, J.J.; Gridley, G.; Hyer, M.; Dwyer, J.T.; Brown, L.M. Flavonoid consumption and esophageal cancer among black and white men in the united states. Int. J. Cancer 2009, 125, 1147-1154. [CrossRef] [PubMed]

23. Frankenfeld, C.L.; Cerhan, J.R.; Cozen, W.; Davis, S.; Schenk, M.; Morton, L.M.; Hartge, P.; Ward, M.H. Dietary flavonoid intake and non-hodgkin lymphoma risk. Am. J. Clin. Nutr. 2008, 87, 1439-1445. [PubMed]

24. Zamora-Ros, R.; Agudo, A.; Lujan-Barroso, L.; Romieu, I.; Ferrari, P.; Knaze, V.; Bueno-de-Mesquita, H.B.; Leenders, M.; Travis, R.C.; Navarro, C.; et al. Dietary flavonoid and lignan intake and gastric adenocarcinoma risk in the European Prospective Investigation into Cancer and Nutrition (EPIC) study. Am. J. Clin. Nutr. 2012, 96, 1398-1408. [CrossRef] [PubMed]

25. Zamora-Ros, R.; Not, C.; Guino, E.; Lujan-Barroso, L.; Garcia, R.M.; Biondo, S.; Salazar, R.; Moreno, V. Association between habitual dietary flavonoid and lignan intake and colorectal cancer in a Spanish case-control study (the bellvitge colorectal cancer study). Cancer Causes Control 2013, 24, 549-557. [CrossRef] [PubMed]

26. Zamora-Ros, R.; Rabassa, M.; Cherubini, A.; Urpi-Sarda, M.; Bandinelli, S.; Ferrucci, L.; Andres-Lacueva, C. High concentrations of a urinary biomarker of polyphenol intake are associated with decreased mortality in older adults. J. Nutr. 2013, 143, 1445-1450. [CrossRef] [PubMed]

27. Tresserra-Rimbau, A.; Rimm, E.B.; Medina-Remon, A.; Martinez-Gonzalez, M.A.; Lopez-Sabater, M.C.; Covas, M.I.; Corella, D.; Salas-Salvado, J.; Gomez-Gracia, E.; Lapetra, J.; et al. Polyphenol intake and mortality risk: A re-analysis of the PREDIMED trial. BMC Med. 2014, 12. [CrossRef] [PubMed]

28. Wang, Z.J.; Ohnaka, K.; Morita, M.; Toyomura, K.; Kono, S.; Ueki, T.; Tanaka, M.; Kakeji, Y.; Maehara, Y.; Okamura, T.; et al. Dietary polyphenols and colorectal cancer risk: The fukuoka colorectal cancer study. World J. Gastroenterol. 2013, 19, 2683-2690. [CrossRef] [PubMed]

29. Fukushima, Y.; Ohie, T.; Yonekawa, Y.; Yonemoto, K.; Aizawa, H.; Mori, Y.; Watanabe, M.; Takeuchi, M.; Hasegawa, M.; Taguchi, C.; et al. Coffee and green tea as a large source of antioxidant polyphenols in the Japanese population. J. Agric. Food Chem. 2009, 57, 1253-1259. [CrossRef] [PubMed]

30. Fukushima, Y.; Tashiro, T.; Kumagai, A.; Ohyanagi, H.; Horiuchi, T.; Takizawa, K.; Sugihara, N.; Kishimoto, Y.; Taguchi, C.; Tani, M.; et al. Coffee and beverages are the major contributors to polyphenol consumption from food and beverages in Japanese middle-aged women. J. Nutr. Sci. 2014, 3, 1-10. [CrossRef] [PubMed]

31. Taguchi, C.; Fukushima, Y.; Kishimoto, Y.; Saita, E.; Suzuki-Sugihara, N.; Yoshida, D.; Kondo, K. Polyphenol intake from beverages in Japan over an 18-year period (1996-2013): Trends by year, age, gender and season. J. Nutr. Sci. Vitaminol. (Tokyo) 2015, 61, 338-344. [CrossRef] [PubMed]

32. Rabassa, M.; Cherubini, A.; Zamora-Ros, R.; Urpi-Sarda, M.; Bandinelli, S.; Ferrucci, L.; Andres-Lacueva, C. Low levels of a urinary biomarker of dietary polyphenol are associated with substantial cognitive decline over a 3-year period in older adults: The invecchiare in CHIANTI study. J. Am. Geriatr. Soc. 2015, 63, 938-946. [CrossRef] [PubMed]

33. Urpi-Sarda, M.; Andres-Lacueva, C.; Rabassa, M.; Ruggiero, C.; Zamora-Ros, R.; Bandinelli, S.; Ferrucci, L.; Cherubini, A. The relationship between urinary total polyphenols and the frailty phenotype in a community-dwelling older population: The InCHIANTI study. J. Gerontol. A Biol. Sci. Med. Sci. 2015, 70, 1141-1147. [CrossRef] [PubMed] 
34. Ministry of Health, labour and Walfare. Dietary Reference Intakes for Japanese 2010; Dai-ichi Shuppan: Tokyo, Japan, 2010; pp. 43-275.

35. Kobayashi, S.; Honda, S.; Murakami, K.; Sasaki, S.; Okubo, H.; Hirota, N.; Notsu, A.; Fukui, M.; Date, C. Both comprehensive and brief self-administered diet history questionnaires satisfactorily rank nutrient intakes in Japanese adults. J. Epidemiol. 2012, 22, 151-159. [CrossRef] [PubMed]

36. Kobayashi, S.; Murakami, K.; Sasaki, S.; Okubo, H.; Hirota, N.; Notsu, A.; Fukui, M.; Date, C. Comparison of relative validity of food group intakes estimated by comprehensive and brief-type self-administered diet history questionnaires against $16 \mathrm{~d}$ dietary records in Japanese adults. Public Health Nutr. 2011, 14, 1200-1211. [CrossRef] [PubMed]

37. Ministry of Health, Labour and Walfare. National Health and Nutrition Survey 2012. Available online: http:/ /www.mhlw.go.jp/bunya/kenkou/eiyou/dl/h24-houkoku-04.pdf (accessed on 15 September 2015).

38. Ovaskainen, M.L.; Torronen, R.; Koponen, J.M.; Sinkko, H.; Hellstrom, J.; Reinivuo, H.; Mattila, P. Dietary intake and major food sources of polyphenols in Finnish adults. J. Nutr. 2008, 138, 562-566. [PubMed]

39. Tresserra-Rimbau, A.; Medina-Remon, A.; Perez-Jimenez, J.; Martinez-Gonzalez, M.A.; Covas, M.I.; Corella, D.; Salas-Salvado, J.; Gomez-Gracia, E.; Lapetra, J.; Aros, F.; et al. Dietary intake and major food sources of polyphenols in a Spanish population at high cardiovascular risk: The PREDIMED study. Nutr. Metab. Cardiovasc. Dis. 2013, 23, 953-959. [CrossRef] [PubMed]

40. Grosso, G.; Stepaniak, U.; Topor-Madry, R.; Szafraniec, K.; Pajak, A. Estimated dietary intake and major food sources of polyphenols in the Polish arm of the HAPIEE study. Nutrition 2014, 30, 1398-1403. [CrossRef] [PubMed]

41. Messina, M.; Nagata, C.; Wu, A.H. Estimated Asian adult soy protein and isoflavone intakes. Nutr. Cancer 2006, 55, 1-12. [CrossRef] [PubMed]

42. Yamori, Y.; Miura, A.; Taira, K. Implications from and for food cultures for cardiovascular diseases: Japanese food, particularly Okinawan diets. Asia Pac. J. Clin. Nutr. 2001, 10, 144-145. [CrossRef] [PubMed]

(C) 2015 by the authors; licensee MDPI, Basel, Switzerland. This article is an open access article distributed under the terms and conditions of the Creative Commons by Attribution (CC-BY) license (http://creativecommons.org/licenses/by/4.0/). 